\title{
Accurate Numerical Method for Pricing Two-Asset American Put Options
}

\author{
Xianbin Wu \\ Junior College, Zhejiang Wanli University, Ningbo 315100, China \\ Correspondence should be addressed to Xianbin Wu; wxb3210@zwu.edu.cn
}

Received 12 October 2012; Accepted 27 November 2012

Academic Editor: Manuel Ruiz Galan

Copyright (C) 2013 Xianbin Wu. This is an open access article distributed under the Creative Commons Attribution License, which permits unrestricted use, distribution, and reproduction in any medium, provided the original work is properly cited.

\begin{abstract}
We develop an accurate finite difference scheme for pricing two-asset American put options. We use the central difference method for space derivatives and the implicit Euler method for the time derivative. Under certain mesh step size limitations, the matrix associated with the discrete operator is an M-matrix, which ensures that the solutions are oscillation-free. We apply the maximum principle to the discrete linear complementarity problem in two mesh sets and derive the error estimates. It is shown that the scheme is second-order convergent with respect to the spatial variables. Numerical results support the theoretical results.
\end{abstract}

\section{Introduction}

An option is a financial instrument that gives the holder the right, but not the obligation, to buy (call option) or to sell (put option) an agreed quantity of a specified asset at a fixed price (exercise or strike price) on (European option) or before (American option) a given date (expiry date). It was shown by Black-Scholes [1] that the value of a European option is governed by a second-order parabolic partial differential equation with respect to the time and the underlying asset price. The value of an American option is determined by a linear complementarity problem involving the Black-Scholes operator $[2,3]$. Since this complementarity problem is, in general, not analytically solvable, numerical approximation to the solution is normally sought in practice.

Various numerical methods have been proposed for the valuation of single-factor American options. Among them, the lattice method [4], the Monte Carlo method [5], the finite difference method [6-8], the finite element method $[9,10]$, and the finite volume method [11-13] are the most popular ones in both practice and research.

Finite difference methods applied to the multifactor American option valuation have also been developed. S. O'Sullivan and C. O'Sullivan [14] presented explicit finite difference methods with an acceleration technique for option pricing. Clarke and Parrott [15] and Oosterlee [16] used finite difference schemes along with a projected full approximation scheme (PFAS) multigrid for pricing American options under stochastic volatility. Ikonen and Toivanen [1719] proposed finite difference methods with componentwise splitting methods on nonuniform grids for pricing American options under stochastic volatility. Hout and Foulon [20] and Zhu and Chen [21] applied finite difference schemes based on the ADI method to price American options under stochastic volatility. Le et al. [22] presented an upwind difference scheme for the valuation of perpetual American put options under stochastic volatility. Yousuf [23] developed an exponential time differencing scheme with a splitting technique for pricing American options under stochastic volatility. Nielsen et al. [24] and Zhang et al. [25] analyzed finite difference schemes with penalty methods for pricing American two-asset options, but their difference methods are first-order convergent.

In part of the domain, the differential operator of the twoasset American option pricing model becomes a convectiondominated operator. The differential operator also contains a second-order mixed derivative term. The classical finite difference methods lead to some off-diagonal elements in the coefficient matrix of the discrete operator due to the dominating first-order derivatives and the mixed derivative. These elements can lead to nonphysical oscillations in the computed solution $[17,18]$. In this paper, we present 
an accurate finite difference scheme for pricing two-asset American options. We use the central difference method for space derivatives and the implicit Euler method for the time derivative. Under certain mesh step size limitations, we obtain a coefficient matrix with an M-matrix property, which ensures that the solutions are oscillation-free. We apply the maximum principle to the discrete linear complementarity problem in two mesh sets and derive the error estimates. We will show that the scheme is second-order convergent with respect to the spatial variables.

The rest of the paper is organized as follows. In the next section, we describe some theoretical results on the continuous complementarity problem for the two-asset American put option pricing model. In Section 3, the discretization method is described. In Section 4, we present a stability and error analysis for the finite difference scheme. In Section 5, numerical experiments are provided to support these theoretical results.

\section{The Continuous Problem}

We consider the following two-asset American put option pricing model $[24,25]$ :

$$
\begin{gathered}
\ell P\left(S_{1}, S_{2}, t\right) \geq 0, \quad S_{1}, S_{2}>0, t \in[0, T), \\
\ell P\left(S_{1}, S_{2}, t\right) \cdot\left[P\left(S_{1}, S_{2}, t\right)-\phi\left(S_{1}, S_{2}\right)\right]=0, \\
S_{1}, S_{2}>0, t \in[0, T), \\
P\left(S_{1}, S_{2}, t\right)-\phi\left(S_{1}, S_{2}\right) \geq 0, \quad S_{1}, S_{2} \geq 0, t \in[0, T], \\
P\left(S_{1}, S_{2}, T\right)=\phi\left(S_{1}, S_{2}\right), \quad S_{1}, S_{2} \geq 0, \\
P\left(0, S_{2}, t\right)=g_{2}\left(S_{2}, t\right), \quad S_{2} \geq 0, t \in[0, T], \\
P\left(S_{1}, 0, t\right)=g_{1}\left(S_{1}, t\right), \quad S_{1} \geq 0, t \in[0, T], \\
\lim _{1} P\left(S_{1}, S_{2}, t\right)=0, \quad S_{2} \geq 0, t \in[0, T], \\
\lim _{S_{2} \rightarrow \infty} P\left(S_{1}, S_{2}, t\right)=0, \quad S_{1} \geq 0, t \in[0, T],
\end{gathered}
$$

where $\ell$ denotes the two-dimensional Black-Scholes operator defined by

$$
\begin{aligned}
\ell P\left(S_{1}, S_{2}, t\right) \equiv & -\frac{\partial P}{\partial t}-\frac{1}{2} \sigma_{1}^{2} S_{1}^{2} \frac{\partial^{2} P}{\partial S_{1}^{2}} \\
& -\rho \sigma_{1} \sigma_{2} S_{1} S_{2} \frac{\partial^{2} P}{\partial S_{1} \partial y}-\frac{1}{2} \sigma_{2}^{2} S_{2}^{2} \frac{\partial^{2} P}{\partial S_{2}^{2}} \\
& -r S_{1} \frac{\partial P}{\partial S_{1}}-r S_{2} \frac{\partial P}{\partial S_{2}}+r P
\end{aligned}
$$

and $\phi\left(S_{1}, S_{2}\right)$ is the final (payoff) condition defined by

$$
\phi\left(S_{1}, S_{2}\right)=\max \left\{E-\left(\alpha_{1} S_{1}+\alpha_{2} S_{2}\right), 0\right\} .
$$

Here, $P$ is the value of the option, $S_{i}$ is the value of the $i$ th underlying asset, $\rho \in[-1,0) \cup(0,1]$ is the correlation of two underlying assets, $r$ is the risk-free interest rate, and $g_{i}(\cdot, \cdot)$ is a given function providing suitable boundary conditions. Typically, $g_{i}(\cdot, \cdot)$ is determined by solving the associated onedimensional American put option problem

$$
\begin{gathered}
\bar{\ell}_{i} g_{i}\left(S_{i}, t\right) \geq 0, \quad S_{i}>0, t \in[0, T), \\
\bar{\ell}_{i} g_{i}\left(S_{i}, t\right) \cdot\left[g_{i}\left(S_{i}, t\right)-\max \left(E-\alpha_{i} S_{i}, 0\right)\right]=0, \\
S_{i}>0, t \in[0, T), \\
g_{i}\left(S_{i}, t\right)-\max \left(E-\alpha_{i} S_{i}, 0\right) \geq 0, \quad S_{i} \geq 0, t \in[0, T], \\
g_{i}\left(S_{i}, T\right)=\max \left(E-\alpha_{i} S_{i}, 0\right), \quad S_{i} \geq 0, \\
g_{i}(0, t)=E, \quad \lim _{S_{i} \rightarrow \infty} g_{i}\left(S_{i}, t\right)=0, \quad t \in[0, T],
\end{gathered}
$$

where $\bar{\ell}_{i}$ denotes the one-dimensional Black-Scholes operator defined by

$$
\bar{\ell}_{i} g_{i}\left(S_{i}, t\right) \equiv-\frac{\partial g_{i}}{\partial t}-\frac{1}{2} \sigma_{i}^{2} S_{i}^{2} \frac{\partial^{2} g_{i}}{\partial S_{i}^{2}}-r S_{i} \frac{\partial g_{i}}{\partial S_{i}}+r g_{i}, \quad i=1,2
$$

Introducing the logarithmic prices $x=\ln S_{1}$ and $y=$ $\ln S_{2}$, the linear complementarity problem (1) is transformed as

$$
\begin{gathered}
L u(x, y, t) \geq 0, \quad(x, y, t) \in \mathbb{R} \times \mathbb{R} \times[0, T), \\
L u(x, y, t) \cdot[u(x, y, t)-\varphi(x, y)]=0, \\
(x, y, t) \in \mathbb{R} \times \mathbb{R} \times[0, T), \\
u(x, y, t)-\varphi(x, y) \geq 0, \quad(x, y, t) \in \mathbb{R} \times \mathbb{R} \times[0, T], \\
u(x, y, T)=\varphi(x, y), \quad(x, y) \in \mathbb{R} \times \mathbb{R}, \\
u(0, y, t)=g_{2}\left(e^{y}, t\right), \quad(y, t) \in \mathbb{R} \times[0, T], \\
u(x, 0, t)=g_{1}\left(e^{x}, t\right), \quad(x, t) \in \mathbb{R} \times[0, T], \\
\lim _{x \rightarrow \infty} u(x, y, t)=0, \quad(y, t) \in \mathbb{R} \times[0, T], \\
\lim _{y \rightarrow \infty} u(x, y, t)=0, \quad(x, t) \in \mathbb{R} \times[0, T],
\end{gathered}
$$

where

$$
\begin{aligned}
& L u(x, y, t) \equiv-\frac{\partial u}{\partial t}-\frac{1}{2} \sigma_{1}^{2} \frac{\partial^{2} u}{\partial x^{2}}-\rho \sigma_{1} \sigma_{2} \frac{\partial^{2} u}{\partial x \partial y}-\frac{1}{2} \sigma_{2}^{2} \frac{\partial^{2} u}{\partial y^{2}} \\
& -\left(r-\frac{1}{2} \sigma_{1}^{2}\right) \frac{\partial u}{\partial x}-\left(r-\frac{1}{2} \sigma_{2}^{2}\right) \frac{\partial u}{\partial y}+r u, \\
& \varphi(x, y)=\max \left\{E-\left(\alpha_{1} e^{x}+\alpha_{2} e^{y}\right), 0\right\} \text {. }
\end{aligned}
$$

For applying the numerical method, we truncate the infinite domain into $\Omega \equiv\left(x_{\min }, x_{\max }\right) \times\left(y_{\min }, y_{\max }\right)$, where the boundaries $x_{\min }, x_{\max }, y_{\min }$, and $y_{\max }$ are chosen so as not to introduce huge errors in the value of the option [26]. Based on Willmott et al's estimate [3] that the upper bound of 
the asset price is typically three or four times the strike price, it is reasonable for us to set $x_{\max }=\ln (4 E)$ and $y_{\max }=\ln (4 E)$. The artificial boundary conditions at $x=x_{\min }$ and $x=x_{\max }$ are chosen to be $u\left(x_{\min }, y, t\right)=g_{2}\left(e^{y}, t\right), u\left(x_{\max }, y, t\right)=0$. The artificial boundary conditions at $y=y_{\min }$ and $y=y_{\max }$ are chosen to be $u\left(x, y_{\min }, t\right)=g_{1}\left(e^{x}, t\right), u\left(x, y_{\max }, t\right)=0$. Therefore, in the rest of this paper, we will consider the following linear complementary problem:

$$
\begin{gathered}
L u(x, y, t) \geq 0, \quad(x, y, t) \in \Omega \times[0, T), \\
L u(x, y, t) \cdot[u(x, y, t)-\varphi(x, y)]=0, \\
(x, y, t) \in \Omega \times[0, T), \\
u(x, y, t)-\varphi(x, y) \geq 0, \quad(x, y, t) \in \Omega \times[0, T], \\
u\left(x x_{\min }, y, t\right)=g_{2}\left(e^{y}, t\right), \quad(y, t) \in\left[y_{\min }, y_{\max }\right] \times[0, T], \\
u\left(x_{\max }, y, t\right)=0, \quad(y, t) \in\left[y_{\min }, y_{\max }\right] \times[0, T], \\
u\left(x, y_{\min }, t\right)=g_{1}\left(e^{x}, t\right), \quad(x, t) \in\left[x_{\min }, x_{\max }\right] \times[0, T], \\
u\left(x, y_{\max }, t\right)=0, \quad(x, t) \in\left[x_{\min }, x_{\max }\right] \times[0, T] .
\end{gathered}
$$

\section{Discretization}

The operator $L$ contains a second-order mixed derivative term. Usual finite difference approximations lead to some positive off-diagonal elements in the matrix associated with the discrete operator due to the mixed derivative, which may lead to nonphysical oscillations in the computed solution. Hence, it is not easy to construct a discretization with good properties and accuracy for problems with mixed derivatives. There are some works dealing with stable difference approximations of mixed derivatives $[27,28]$. In this paper, we present an accurate finite difference scheme to discretize the operator $L$. We use the technique of [22] to give the mesh step size limitation, which guarantees that the coefficient matrix corresponding to the discrete operator is an $M$-matrix.

The discretization is performed using a uniform mesh $\Omega^{N, M, K}$ for the computational domain $\Omega \times[0, T]$. The mesh steps to the $x$ direction, $y$ direction, and $t$ direction are denoted by $\Delta x=\left(x_{\max }-x_{\min }\right) / N, \Delta y=\left(y_{\max }-y_{\min }\right) / M$, and $\Delta t=T / K$. The mesh point values of the finite difference approximation are denoted by

$$
\begin{aligned}
& U_{i, j}^{k} \approx u\left(x_{i}, y_{j}, t_{k}\right) \quad \text { for } i=0,1, \ldots, N ; \\
& \quad j=0,1, \ldots, M ; k=0,1, \ldots, K .
\end{aligned}
$$

We discretize the differential operator $L$ using the central difference scheme on the previous uniform mesh. We set

$$
\begin{aligned}
L^{N, M, K} & U_{i, j}^{k} \\
\equiv & -D_{t}^{+} U_{i, j}^{k}-\frac{1}{2} \sigma_{1}^{2} \delta_{x}^{2} U_{i, j}^{k} \\
& -\sigma_{1} \sigma_{2}\left(\tilde{\rho}^{+} \delta_{x y}^{+} U_{i, j}^{k}+\tilde{\rho}^{-} \delta_{x y}^{-} U_{i, j}^{k}\right)-\frac{1}{2} \sigma_{2}^{2} \delta_{y}^{2} U_{i, j}^{k} \\
& -\left(r-\frac{1}{2} \sigma_{1}^{2}\right) D_{x} U_{i, j}^{k}-\left(r-\frac{1}{2} \sigma_{2}^{2}\right) D_{y} U_{i, j}^{k}+r U_{i, j}^{k},
\end{aligned}
$$

where

$$
\begin{aligned}
& \delta_{x}^{2} U_{i, j}^{k}=\frac{D_{x}^{+}-D_{x}^{-}}{\Delta x} U_{i, j}^{k}, \quad \delta_{y}^{2} U_{i, j}^{k}=\frac{D_{y}^{+}-D_{y}^{-}}{\Delta y} U_{i, j}^{k}, \\
& \delta_{x y}^{+} U_{i, j}^{k}=\frac{D_{x}^{+} D_{y}^{+}+D_{x}^{-} D_{y}^{-}}{2} U_{i, j}^{k} \text {, } \\
& \delta_{x y}^{-} U_{i, j}^{k}=\frac{D_{x}^{+} D_{y}^{-}+D_{x}^{-} D_{y}^{+}}{2} U_{i, j}^{k} \text {, } \\
& D_{x}^{+} U_{i, j}^{k}=\frac{U_{i+1, j}^{k}-U_{i, j}^{k}}{\Delta x}, \quad D_{y}^{+} U_{i, j}^{k}=\frac{U_{i, j+1}^{k}-U_{i, j}^{k}}{\Delta y}, \\
& D_{x}^{-} U_{i, j}^{k}=\frac{U_{i, j}^{k}-U_{i-1, j}^{k}}{\Delta x}, \quad D_{y}^{-} U_{i, j}^{k}=\frac{U_{i, j}^{k}-U_{i, j-1}^{k}}{\Delta y}, \\
& D_{x} U_{i, j}^{k}=\frac{U_{i+1, j}^{k}-U_{i-1, j}^{k}}{2 \Delta x}, \quad D_{y} U_{i, j}^{k}=\frac{U_{i, j+1}^{k}-U_{i, j-1}^{k}}{2 \Delta y}, \\
& D_{t}^{+} U_{i, j}^{k}=\frac{U_{i, j}^{k+1}-U_{i, j}^{k}}{\Delta t}, \quad \tilde{\rho}^{ \pm}=\frac{1}{2}[\rho \pm|\rho|] .
\end{aligned}
$$

Denote

$$
\begin{aligned}
& \bar{\Omega}_{h}=\{(i, j, k) \mid 0 \leq i \leq N, 0 \leq j \leq M, 0 \leq k \leq K\}, \\
& \widetilde{\Omega}_{h}=\{(i, j, k) \mid 1 \leq i \leq N-1,1 \leq j \leq M-1, \\
& \quad 1 \leq k \leq K-1\}, \\
& \partial \Omega_{h}=\bar{\Omega}_{h} \backslash \widetilde{\Omega}_{h} .
\end{aligned}
$$

Thus, we apply the central difference scheme on the uniform mesh to approximate the parabolic complementarity problem (8) as follows:

$$
\begin{gathered}
L^{N, M, K} U_{i, j}^{k} \geq 0, \quad(i, j, k) \in \widetilde{\Omega}_{h}, \\
U_{i, j}^{k}-\varphi_{i, j} \geq 0, \quad(i, j, k) \in \widetilde{\Omega}_{h}, \\
L^{N, M, K} U_{i, j}^{k} \cdot\left[U_{i, j}^{k}-\varphi_{i, j}\right]=0, \quad(i, j, k) \in \widetilde{\Omega}_{h}, \\
U_{i, j}^{K}=\varphi_{i, j}, \quad 0 \leq i \leq N, \quad 0 \leq j \leq M, \\
U_{0, j}^{k}=\left(g_{2}\right)_{j}^{k}, \quad U_{N, j}^{k}=0, \quad 0 \leq j \leq M, 0 \leq k<K, \\
U_{i, 0}^{k}=\left(g_{1}\right)_{i}^{k}, \quad U_{i, M}^{k}=0, \quad 0<i<N, \quad 0 \leq k<K .
\end{gathered}
$$


Here, $\left(g_{1}\right)_{i}^{k}$ and $\left(g_{2}\right)_{j}^{k}$ are discrete approximates of $g_{1}\left(e^{x}\right.$, $t)$ and $g_{2}\left(e^{y}, t\right)$, respectively. Hence, $\left(g_{1}\right)_{i}^{k}$ and $\left(g_{2}\right)_{j}^{k}$ can be obtained by solving the corresponding one-dimensional Black-Scholes equations [29]. In the next section, we will prove that the system matrix corresponding to the discrete operator $L^{N, M, K}$ is an M-matrix. Hence, from the uniqueness theorem of Goeleven [30], we can obtain that there exists a unique solution $U$ for the previous linear complementarity problem (13).

\section{Analysis of the Method}

First, we give the stability analysis for the difference scheme (13).

Lemma 1. If mesh steps satisfy the inequalities

$$
\begin{gathered}
\Delta x \leq \frac{\sigma_{1}^{2}}{\left|2 r-\sigma_{1}^{2}\right|}, \quad \Delta y \leq \frac{\sigma_{2}^{2}}{\left|2 r-\sigma_{2}^{2}\right|}, \\
\quad \frac{2|\rho| \sigma_{1}}{\sigma_{2}} \leq \frac{\Delta x}{\Delta y} \leq \frac{2 \sigma_{1}}{|\rho| \sigma_{2}},
\end{gathered}
$$

then the system matrix corresponding to the discrete operator $L^{N, M, K}$ is an $M$-matrix.

Proof. The difference operator $L^{N, M, K}$ can be written as follows:

$$
\begin{aligned}
L^{N, M,}, & U_{i, j}^{k} \\
= & -\frac{\tilde{\rho}^{+} \sigma_{1} \sigma_{2}}{2 \Delta x \Delta y} U_{i-1, j-1}^{k} \\
+ & {\left[\sigma_{1} \sigma_{2} \frac{\tilde{\rho}^{+}-\tilde{\rho}^{-}}{2 \Delta x \Delta y}-\frac{\sigma_{2}^{2}}{2(\Delta y)^{2}}+\frac{r-(1 / 2) \sigma_{2}^{2}}{2 \Delta y}\right] U_{i, j-1}^{k} } \\
+ & \frac{\tilde{\rho}^{-} \sigma_{1} \sigma_{2}}{2 \Delta x \Delta y} U_{i+1, j-1}^{k} \\
+ & {\left[\sigma_{1} \sigma_{2} \frac{\tilde{\rho}^{+}-\tilde{\rho}^{-}}{2 \Delta x \Delta y}-\frac{\sigma_{1}^{2}}{2(\Delta x)^{2}}+\frac{r-(1 / 2) \sigma_{1}^{2}}{2 \Delta x}\right] U_{i-1, j}^{k} } \\
+ & {\left[\frac{1}{\Delta t}+\frac{\sigma_{1}^{2}}{(\Delta x)^{2}}-\sigma_{1} \sigma_{2} \frac{\tilde{\rho}^{+}-\tilde{\rho}^{-}}{\Delta x \Delta y}+\frac{\sigma_{2}^{2}}{(\Delta y)^{2}}+r\right] U_{i, j}^{k} } \\
+ & \frac{\tilde{\rho}^{+} \sigma_{1} \sigma_{2}}{2 \Delta x \Delta y} U_{i+1, j+1}^{k}-\frac{1}{\Delta t} U_{i, j}^{k+1} \cdot \\
+ & {\left[-\frac{\sigma_{1}^{2}}{2(\Delta x)^{2}}+\sigma_{1} \sigma_{2} \frac{\tilde{\rho}^{+}-\tilde{\rho}^{-}}{2 \Delta x \Delta y}-\frac{r-(1 / 2) \sigma_{1}^{2}}{2 \Delta x}\right] U_{i+1, j}^{k} } \\
+ & \frac{\tilde{\rho}^{-} \sigma_{1} \sigma_{2}}{2 \Delta x \Delta y} U_{i-1, j+1}^{k} \\
+ & {\left[\sigma_{1} \sigma_{2} \frac{\tilde{\rho}^{+}-\tilde{\rho}^{-}}{2 \Delta x \Delta y}-\frac{\sigma_{2}^{2}}{2(\Delta y)^{2}}-\frac{r-(1 / 2) \sigma_{2}^{2}}{2 \Delta y}\right] U_{i, j+1}^{k} }
\end{aligned}
$$

The coefficient of $U_{i, j}$ in the previous expression (which corresponds to the diagonal of the system matrix) is positive since

$$
\frac{\sigma_{1}^{2}}{(\Delta x)^{2}}-\sigma_{1} \sigma_{2} \frac{\tilde{\rho}^{+}-\tilde{\rho}^{-}}{\Delta x \Delta y}+\frac{\sigma_{2}^{2}}{(\Delta y)^{2}} \geq 0 .
$$

All the coefficients of the other $U$ in the previous expression (which correspond to off-diagonal elements in the system matrix) will be nonpositive once the following inequalities are satisfied:

$$
\begin{array}{ll}
\frac{\sigma_{1}^{2}}{4(\Delta x)^{2}}-\frac{\left|r-(1 / 2) \sigma_{1}^{2}\right|}{2 \Delta x} \geq 0, & \frac{\sigma_{2}^{2}}{4(\Delta y)^{2}}-\frac{\left|r-(1 / 2) \sigma_{2}^{2}\right|}{2 \Delta y} \geq 0, \\
\sigma_{1} \sigma_{2} \frac{\tilde{\rho}^{+}-\tilde{\rho}^{-}}{2 \Delta x \Delta y}-\frac{\sigma_{2}^{2}}{4(\Delta y)^{2}} \leq 0, & \sigma_{1} \sigma_{2} \frac{\tilde{\rho}^{+}-\tilde{\rho}^{-}}{2 \Delta x \Delta y}-\frac{\sigma_{1}^{2}}{4(\Delta x)^{2}} \leq 0 .
\end{array}
$$

Together, they require that the following inequalities hold:

$$
\begin{gathered}
\Delta x \leq \frac{\sigma_{1}^{2}}{\left|2 r-\sigma_{1}^{2}\right|}, \quad \Delta y \leq \frac{\sigma_{2}^{2}}{\left|2 r-\sigma_{2}^{2}\right|}, \\
\frac{2 \sigma_{1}\left(\tilde{\rho}^{+}-\tilde{\rho}^{-}\right)}{\sigma_{2}} \leq \frac{\Delta x}{\Delta y} \leq \frac{2 \sigma_{1}}{\sigma_{2}\left(\tilde{\rho}^{+}-\tilde{\rho}^{-}\right)},
\end{gathered}
$$

which are (14) and (15), respectively. Thus, we have shown that the system matrix, corresponding to the discrete operator $L^{N, M, K}$ is an $M$-matrix and the result follows.

There are only few error estimates for the direct application of finite difference method to linear complementarity problems. Here, we apply the maximum principle to the linear complementarity problem (13) in two mesh sets and derive the error estimates [29, 31].

By using Taylor's formula, we can easily obtain the following truncation error estimate.

Lemma 2. Let $u(x, y, t)$ be a smooth function defined on $\Omega^{N, M, K}$. Then the truncation error of the difference scheme (10) satisfies

$$
\left|L^{N, M, K} u_{i, j}^{k}-L u_{i, j}^{k}\right|=O\left((\Delta x)^{2}+(\Delta y)^{2}+\Delta x \Delta y+\Delta t\right),
$$

for all $(i, j, k) \in \widetilde{\Omega}_{h}$.

Now we can derive our main result for the difference scheme.

Theorem 3. Let $u(x, y, t)$ be the solution of the problem (8) and let $U_{i, j}^{k}$ be the solution of the problem (13). If mesh steps satisfy conditions (14) and (15), the difference scheme (13) satisfies the following error estimate:

$$
\max _{(i, j, k) \in \bar{\Omega}_{h}}\left|u\left(x_{i}, y_{j}, t_{k}\right)-U_{i, j}^{k}\right| \leq C\left[(\Delta x)^{2}+(\Delta y)^{2}+\Delta x \Delta y+\Delta t\right],
$$

where $C$ is a constant independent of $\Delta x, \Delta y$, and $\Delta t$. 
Proof. Denote

$$
\begin{gathered}
\Omega^{(1)}=\left\{(i, j, k) \in \widetilde{\Omega}_{h} \mid u\left(x_{i}, y_{j}, t_{k}\right)=\varphi\left(x_{i}, y_{j}\right)\right\}, \\
\Omega^{(2)}=\widetilde{\Omega}_{h} \backslash \Omega^{(1)} .
\end{gathered}
$$

From (8), we have the result

$$
\begin{aligned}
& \operatorname{Lu}\left(x_{i}, y_{j}, t_{k}\right) \geq 0, \quad(i, j, k) \in \Omega^{(1)}, \\
& \operatorname{Lu}\left(x_{i}, y_{j}, t_{k}\right)=0, \quad(i, j, k) \in \Omega^{(2)} .
\end{aligned}
$$

Denote

$$
\begin{gathered}
\Omega_{h}^{(1)}=\left\{(i, j) \in \widetilde{\Omega}_{h} \mid U_{i, j}^{k}=\varphi\left(x_{i}, y_{j}\right)\right\}, \\
\Omega_{h}^{(2)}=\widetilde{\Omega}_{h} \backslash \Omega_{h}^{(1)} .
\end{gathered}
$$

Obviously,

$$
L^{N, M, K} U_{i, j}^{k}=0, \quad(i, j, k) \in \Omega_{h}^{(2)} .
$$

Define the function on $\widetilde{\Omega}_{h}$ by

$$
W_{i, j}^{k}=C\left[(\Delta x)^{2}+(\Delta y)^{2}+\Delta x \Delta y+\Delta t\right]>0,
$$

where $C$ is a sufficiently large constant.

For $(i, j, k) \in \Omega_{h}^{(2)}$, by the fact that $L u\left(x_{i}, y_{j}, t_{k}\right) \geq 0,(25)$, (26), and Lemma 2, we obtain

$$
\begin{aligned}
L^{N, M, K} & \left(u\left(x_{i}, y_{j}, t_{k}\right)-U_{i, j}^{k}+W_{i, j}^{k}\right) \\
= & L^{N, M, K} u\left(x_{i}, y_{j}, t_{k}\right)+L^{N, M, K} W_{i, j}^{k} \\
= & {\left[L^{N, M, K} u\left(x_{i}, y_{j}, t_{k}\right)-L u\left(x_{i}, y_{j}, t_{k}\right)+L^{N, M, K} W_{i, j}^{k}\right] } \\
& +L u\left(x_{i}, y_{j}, t_{k}\right) \geq 0 .
\end{aligned}
$$

On the "boundary" of $\Omega_{h}^{(2)}$, the nodes $(i, j, k) \in \Omega_{h}^{(1)}$, so $U_{i, j}^{k}=$ $\varphi\left(x_{i}, y_{j}\right)$, but $u\left(x_{i}, y_{j}, t_{k}\right) \geq \varphi\left(x_{i}, y_{j}\right)$, therefore

$$
u\left(x_{i}, y_{j}, t_{k}\right)-U_{i, j}^{k}+W_{i, j}^{k}=u\left(x_{i}, y_{j}, t_{k}\right)-\varphi\left(x_{i}, y_{j}\right)+W_{i, j}^{k} \geq 0,
$$

and the nodes $(i, j, k) \in \partial \Omega_{h}$,

$$
u\left(x_{i}, y_{j}, t_{k}\right)-U_{i, j}^{k}+W_{i, j}^{k}=W_{i, j}^{k} \geq 0 .
$$

Applying the maximum principle to $\Omega_{h}^{(2)}$, we get

$$
u\left(x_{i}, y_{j}, t_{k}\right)-U_{i, j}^{k}+W_{i, j}^{k} \geq 0, \quad(i, j, k) \in \Omega_{h}^{(2)} .
$$

Thus,

$$
u\left(x_{i}, y_{j}, t_{k}\right)-U_{i, j}^{k}+W_{i, j}^{k} \geq 0, \quad(i, j, k) \in \bar{\Omega}_{h} .
$$

For $(i, j, k) \in \Omega^{(2)}, L u\left(x_{i}, y_{j}, t_{k}\right)=0$, but $L^{N, M, K} U_{i, j}^{k} \geq 0$, thus,

$$
\begin{aligned}
L^{N, M, K} & \left(u\left(x_{i}, y_{j}, t_{k}\right)-U_{i, j}^{k}-W_{i, j}^{k}\right) \\
= & {\left[L^{N, M, K} u\left(x_{i}, y_{j}, t_{k}\right)-L u\left(x_{i}, y_{j}, t_{k}\right)-L^{N, M, K} W_{i, j}^{k}\right] } \\
& -L^{N, M, K} U_{i, j}^{k} \leq 0 .
\end{aligned}
$$

On the "boundary" of $\Omega^{(2)}$, the nodes $(i, j, k) \in \Omega^{(1)}$, so $u\left(x_{i}, y_{j}, t_{k}\right)=\varphi\left(x_{i}, y_{j}\right)$, but $U_{i, j}^{k} \geq \varphi\left(x_{i}, y_{j}\right)$, therefore

$$
u\left(x_{i}, y_{j}, t_{k}\right)-U_{i, j}^{k}-W_{i, j}^{k}=\varphi\left(x_{i}, y_{j}\right)-U_{i, j}^{k}-W_{i, j}^{k} \leq 0,
$$

and the nodes $(i, j, k) \in \partial \Omega_{h}$,

$$
u\left(x_{i}, y_{j}, t_{k}\right)-U_{i, j}^{k}-W_{i, j}^{k}=-W_{i, j}^{k} \leq 0 .
$$

Applying the maximum principle to $\Omega^{(2)}$, we get

$$
u\left(x_{i}, y_{j}, t_{k}\right)-U_{i, j}^{k}-W_{i, j}^{k} \leq 0, \quad(i, j, k) \in \Omega^{(2)} .
$$

Thus,

$$
u\left(x_{i}, y_{j}, t_{k}\right)-U_{i, j}^{k}-W_{i, j}^{k} \leq 0, \quad(i, j, k) \in \bar{\Omega}_{h} .
$$

From (31) and (36), we obtain

$$
\begin{aligned}
& \max _{(i, j, k) \in \bar{\Omega}_{h}}\left|u\left(x_{i}, y_{j}, t_{k}\right)-U_{i, j}^{k}\right| \\
& \quad \leq \max _{(i, j, k) \in \bar{\Omega}_{h}} W_{i, j}^{k} \leq C\left[(\Delta x)^{2}+(\Delta y)^{2}+\Delta x \Delta y+\Delta t\right],
\end{aligned}
$$

where $C$ is a sufficiently large constant. From this we complete the proof.

\section{Numerical Experiments}

In this section, we verify experimentally the theoretical results obtained in the preceding section. Errors and convergence rates for the second-order finite difference scheme are presented for two test problems.

Test 1. American put option with parameters: $T=1, r=0.1$, $\sigma_{1}=0.4, \sigma_{2}=0.5, \rho=0.5, x_{\min }=-\ln (30), x_{\max }=\ln (30)$, $y_{\min }=-\ln (40), y_{\max }=\ln (40), \alpha_{1}=0.3, \alpha_{2}=0.7$, and $E=$ 10

Test 2. American put option with parameters: $T=1, r=$ $0.08, \sigma_{1}=0.3, \sigma_{2}=0.4, \rho=-0.6, x_{\min }=-\ln (30), x_{\max }=$ $\ln (30), y_{\min }=-\ln (40), y_{\max }=\ln (40), \alpha_{1}=0.3, \alpha_{2}=0.7$, and $E=10$.

To solve the linear inequality system (13), we use the projection scheme used in [32, page 433]. Since mesh steps need to satisfy conditions (14) and (15), we choose the number of mesh steps in the $y$ direction

$$
M=\left[\frac{\left(2|\rho| \sigma_{1} / \sigma_{2}+2 \sigma_{1} /|\rho| \sigma_{2}\right) N}{2\left(x_{\max }-x_{\min }\right)}\right],
$$


TABLE 1: Numerical results for Test 1.

\begin{tabular}{cccc}
\hline$K$ & $N$ & Error & Rate \\
\hline \multirow{3}{*}{128} & 6 & $1.8124 e-1$ & - \\
& 12 & $5.1852 e-2$ & 1.805 \\
& 24 & $1.4761 e-2$ & 1.813 \\
& 48 & $4.1312 e-3$ & 1.837 \\
\hline
\end{tabular}

TABLE 2: Numerical results for Test 2.

\begin{tabular}{cccc}
\hline$K$ & $N$ & Error & Rate \\
\hline \multirow{4}{*}{128} & 6 & $1.2256 e-1$ & - \\
& 12 & $3.3776 e-2$ & 1.859 \\
& 24 & $9.2571 e-3$ & 1.867 \\
& 48 & $2.5124 e-3$ & 1.882 \\
\hline
\end{tabular}

where $N$ is the number of mesh steps in the $x$ direction. The exact solutions of the test problems are not available. Therefore, we use the double mesh principle to estimate the errors and compute the experiment convergence rates in our computed solution. We measure the accuracy in the discrete maximum norm

$$
e^{N, M, K}=\max _{i, j, k}\left|U_{i, j, k}^{N, M, K}-U_{i, j, k}^{2 N, 2 M, K}\right|,
$$

and the convergence rate

$$
R^{N, M, K}=\log _{2}\left(\frac{e^{N, M, K}}{e^{2 N, 2 M, K}}\right) .
$$

The error estimates and convergence rates in our computed solutions of Tests 1 and 2 are listed in Tables 1 and 2, respectively. From Tables 1 and 2 , we see that $e^{N, M, K} / e^{2 N, 2 M, K}$ is close to 4 for sufficiently large $K$, which supports the convergence estimate of Theorem 3 . However, the numerical results of Nielsen et al. [24] and Zhang et al. [25] verify that their schemes are only first-order convergent. Hence, our scheme is more accurate.

\section{Acknowledgments}

The authors would like to thank the anonymous referees for several suggestions for the improvement of this paper. The work was supported by Zhejiang Province Natural Science Foundation of China (Grant no. Y2111160).

\section{References}

[1] F. Black and M. Scholes, "The pricing of options and corporate liabilities," Journal of Political Economy, vol. 81, no. 3, pp. 637654, 1973.

[2] J. Huang and J. S. Pang, "Option pricing and linear complementarity," Journal of Computational Finance, vol. 2, no. 3, pp. 31-60, 1998.

[3] P. Wilmott, J. Dewynne, and S. Howison, Option Pricing: Mathematical Models and Computation, Oxford Financial Press, Oxford, UK, 1993.
[4] J. C. Cox, S. A. Ross, and M. Rubinstein, "Option pricing: a simplified approach," Journal of Financial Economics, vol. 7, no. 3, pp. 229-263, 1979.

[5] A. Ibáñez and F. Zapatero, "Monte Carlo valuation of American options through computation of the optimal exercise frontier," Journal of Financial and Quantitative Analysis, vol. 39, no. 2, pp. 253-275, 2004.

[6] C. Vázquez, "An upwind numerical approach for an American and European option pricing model," Applied Mathematics and Computation, vol. 97, no. 2-3, pp. 273-286, 1998.

[7] L. Wu and Y. K. Kwok, "A front-xing nite dierence method for the valuation of American options," Journal of Financial Engineering, vol. 6, no. 2, pp. 83-97, 1997.

[8] J. Zhao, M. Davison, and R. M. Corless, "Compact finite difference method for American option pricing," Journal of Computational and Applied Mathematics, vol. 206, no. 1, pp. 306-321, 2007.

[9] P. A. Forsyth, K. R. Vetzal, and R. Zvan, "A nite element approach to the pricing of discrete lookbacks with stochastic volatility," Applied Mathematical Finance, vol. 6, no. 2, pp. 87106, 1999.

[10] R. Zvan, P. A. Forsyth, and K. R. Vetzal, "A general nite element approach for PDE option pricing models," University of Waterloo, Canada, 1998.

[11] L. Angermann and S. Wang, "Convergence of a fitted finite volume method for the penalized Black-Scholes equation governing European and American option pricing," Numerische Mathematik, vol. 106, no. 1, pp. 1-40, 2007.

[12] S. Wang, X. Q. Yang, and K. L. Teo, "Power penalty method for a linear complementarity problem arising from American option valuation," Journal of Optimization Theory and Applications, vol. 129, no. 2, pp. 227-254, 2006.

[13] R. Zvan, P. A. Forsyth, and K. R. Vetzal, "A finite volume approach for contingent claims valuation," IMA Journal of Numerical Analysis, vol. 21, no. 3, pp. 703-731, 2001.

[14] S. O'Sullivan and C. O'Sullivan, "On the acceleration of explicit finite difference methods for option pricing," Quantitative Finance, vol. 11, no. 8, pp. 1177-1191, 2011.

[15] N. Clarke and K. Parrott, "Multigrid for American option pricing with stochastic volatility," Applied Mathematics Finance, vol. 6, no. 3, pp. 177-195, 1999.

[16] C. W. Oosterlee, "On multigrid for linear complementarity problems with application to American-style options," Electronic Transactions on Numerical Analysis, vol. 15, pp. 165-185, 2003.

[17] S. Ikonen and J. Toivanen, "Componentwise splitting methods for pricing American options under stochastic volatility," International Journal of Theoretical and Applied Finance, vol. 10, no. 2, pp. 331-361, 2007.

[18] S. Ikonen and J. Toivanen, "Efficient numerical methods for pricing American options under stochastic volatility," Numerical Methods for Partial Differential Equations, vol. 24, no. 1, pp. 104-126, 2008.

[19] S. Ikonen and J. Toivanen, "Operator splitting methods for pricing American options under stochastic volatility," Numerische Mathematik, vol. 113, no. 2, pp. 299-324, 2009.

[20] K. J. I. Hout and S. Foulon, "ADI finite difference schemes for option pricing in the Heston model with correlation," International Journal of Numerical Analysis and Modeling, vol. 7, no. 2, pp. 303-320, 2010. 
[21] S.-P. Zhu and W.-T. Chen, "A predictor-corrector scheme based on the ADI method for pricing American puts with stochastic volatility," Computers \& Mathematics with Applications, vol. 62, no. 1, pp. 1-26, 2011.

[22] A. Le, Z. Cen, and A. Xu, "A robust upwind difference scheme for pricing perpetual American put options under stochastic volatility," International Journal of Computer Mathematics, vol. 89, no. 9, pp. 1135-1144, 2012.

[23] M. Yousuf, "Efficient $L$-stable method for parabolic problems with application to pricing American options under stochastic volatility," Applied Mathematics and Computation, vol. 213, no. 1, pp. 121-136, 2009.

[24] B. F. Nielsen, O. Skavhaug, and A. Tveito, "Penalty methods for the numerical solution of American multi-asset option problems," Journal of Computational and Applied Mathematics, vol. 222, no. 1, pp. 3-16, 2008.

[25] K. Zhang, S. Wang, X. Q. Yang, and K. L. Teo, "A power penalty approach to numerical solutions of two-asset American options," Numerical Mathematics. Theory, Methods and Applications, vol. 2, no. 2, pp. 202-223, 2009.

[26] R. Kangro and R. Nicolaides, "Far field boundary conditions for Black-Scholes equations," SIAM Journal on Numerical Analysis, vol. 38, no. 4, pp. 1357-1368, 2000.

[27] P. Matus and I. Rybak, "Difference schemes for elliptic equations with mixed derivatives," Computational Methods in Applied Mathematics, vol. 4, no. 4, pp. 494-505, 2004.

[28] I. V. Rybak, "Monotone and conservative difference schemes for elliptic equations with mixed derivatives," Mathematical Modelling and Analysis, vol. 9, no. 2, pp. 169-178, 2004.

[29] Z. Cen and A. Le, "A robust finite difference scheme for pricing American put options with singularity-separating method," Numerical Algorithms, vol. 53, no. 4, pp. 497-510, 2010.

[30] D. Goeleven, "A uniqueness theorem for the generalized-order linear complementary problem associated with $M$-matrices," Linear Algebra and Its Applications, vol. 235, pp. 221-227, 1996.

[31] X. Cheng and L. Xue, "On the error estimate of finite difference method for the obstacle problem," Applied Mathematics and Computation, vol. 183, no. 1, pp. 416-422, 2006.

[32] R. Glowinski, J. L. Lions, and T. Trémolières, Numerical Analysis of Variational Inequality, North-Holland, Amsterdam, The Netherlands, 1984. 


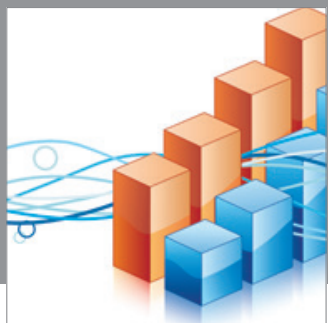

Advances in

Operations Research

mansans

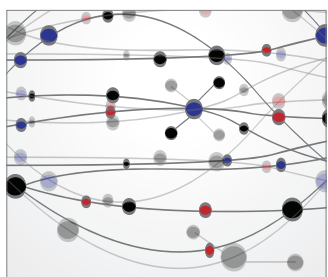

The Scientific World Journal
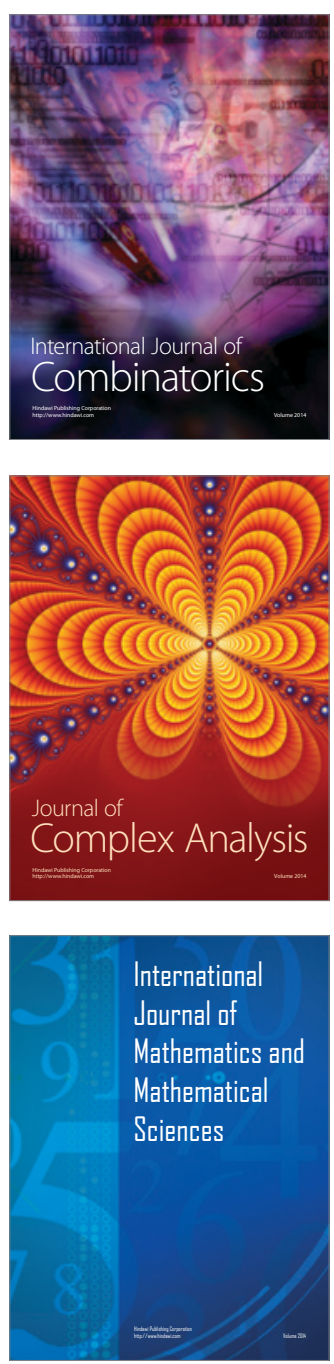
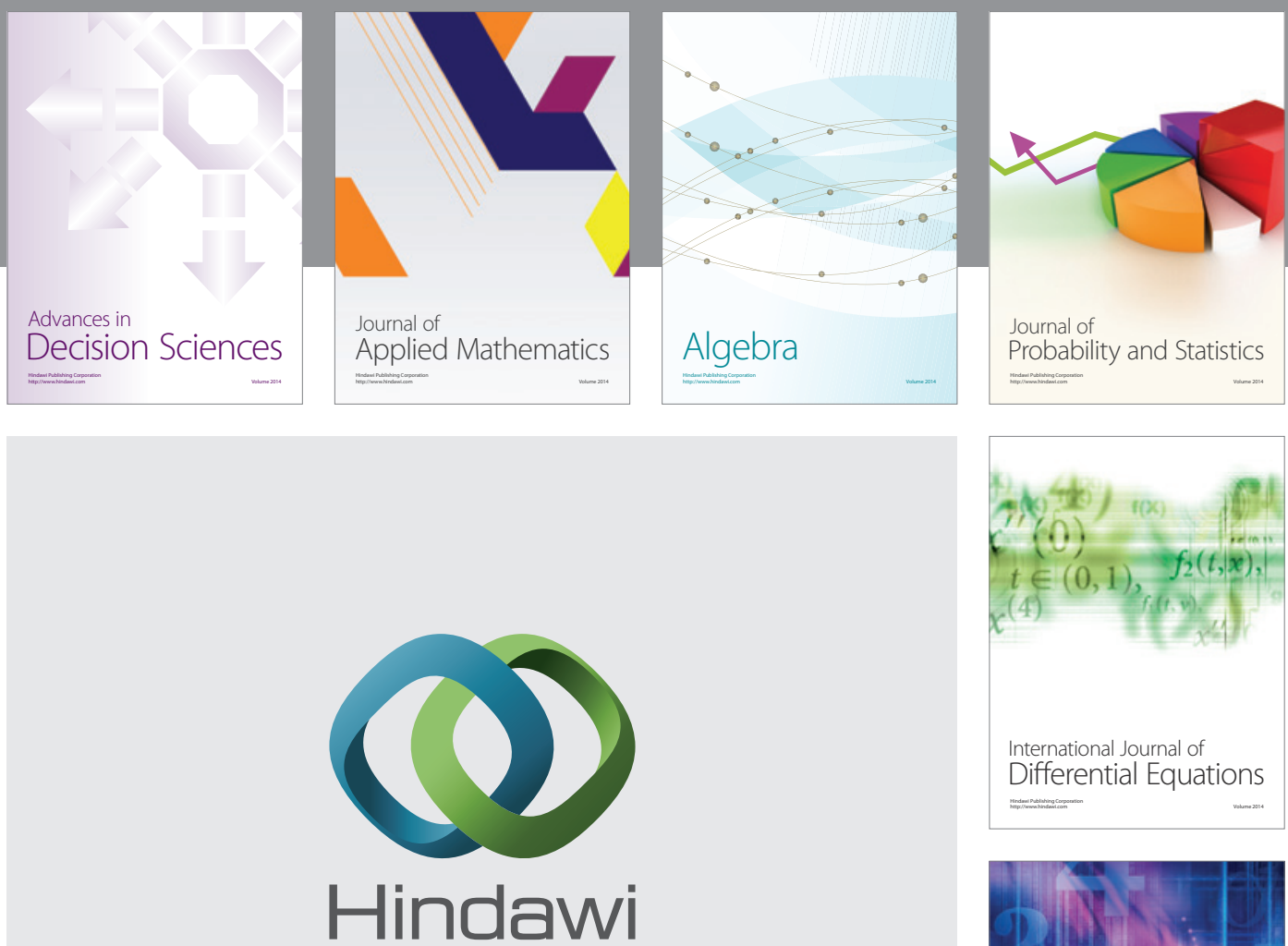

Submit your manuscripts at http://www.hindawi.com
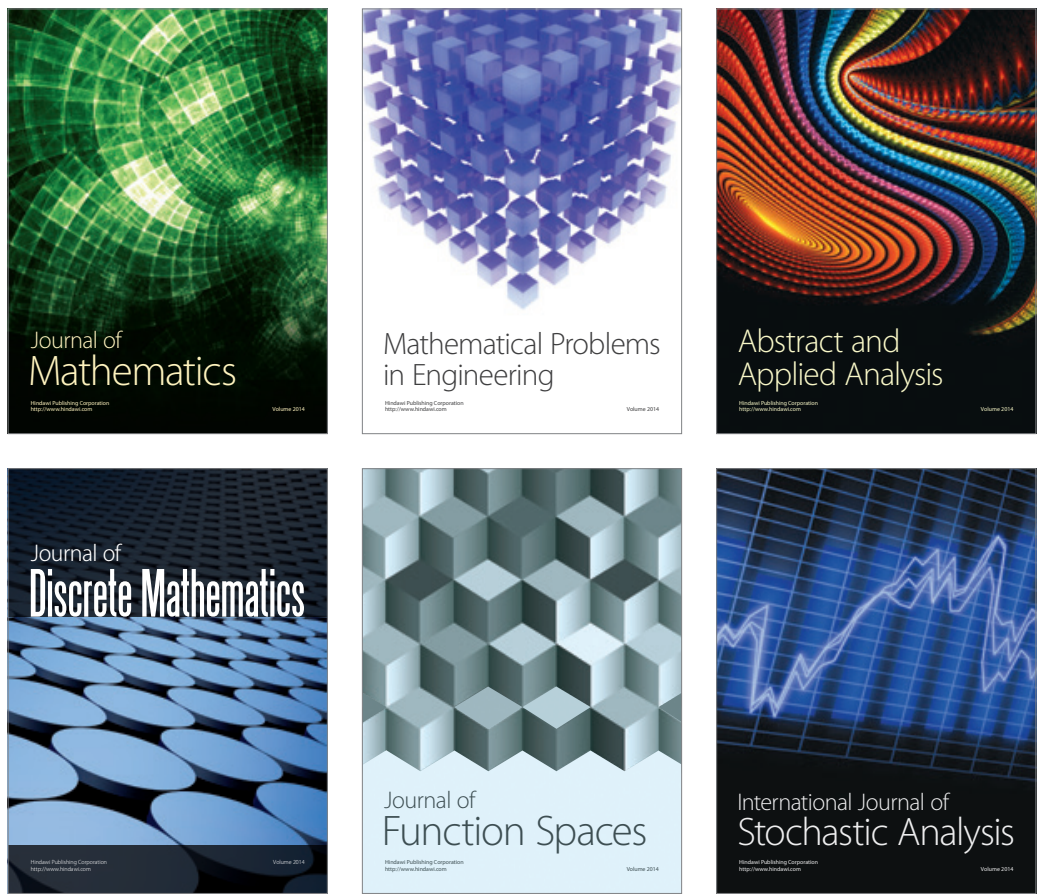

Journal of

Function Spaces

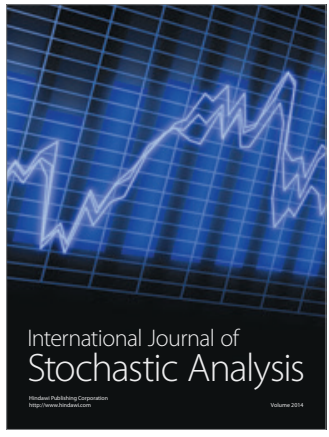

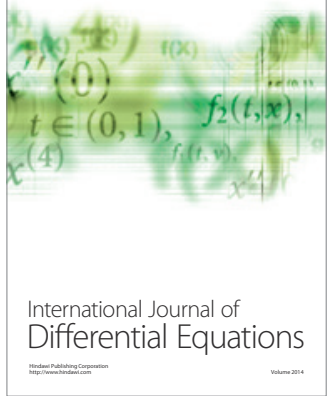
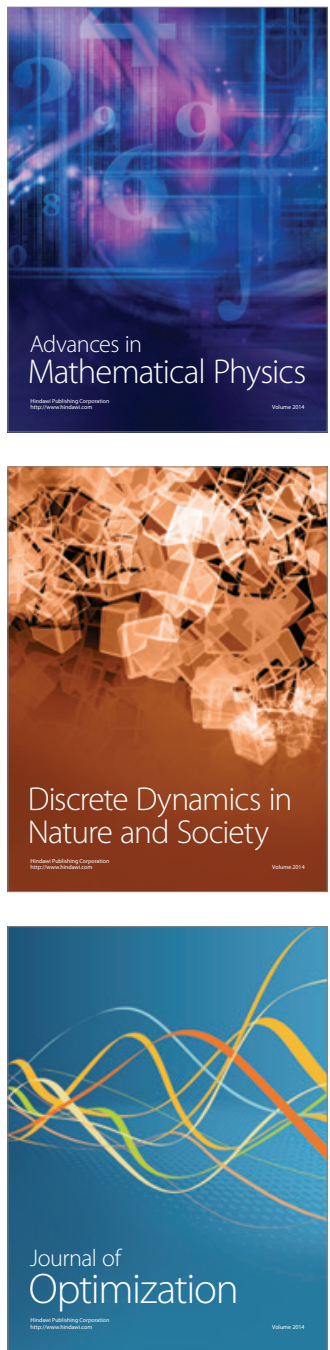